\title{
Liver Fibrosis Helps to Distinguish Autoimmune Hepatitis from DILI with Autoimmune Features: A Review of Twenty Cases
}

\author{
Christopher A. Febres-Aldana*1, Sarah Alghamdi ${ }^{1}$, Kritika Krishnamurthy ${ }^{1}$ \\ and Robert J. Poppiti ${ }^{12}$ \\ ${ }^{1}$ Arkadi M. Rywlin M.D. Department of Pathology and Laboratory Medicine, Mount Sinai Medical Center, Miami Beach, FL, USA; \\ ${ }^{2}$ Herbert Wertheim College of Medicine. Florida International University, Miami, FL, USA
}

\begin{abstract}
Background and Aims: Drug-induced liver injury with autoimmune features (AI-DILI) mimics the clinical presentation, and laboratory and pathologic features of idiopathic autoimmune hepatitis (AIH). We aimed to identify histopathologic hallmarks to differentiate these entities. Methods: All liver biopsies archived for the past 10 years were reviewed retrospectively to identify cases of recently detected liver injury associated with predominantly lymphoplasmacytic interphase hepatitis, positive markers for liver autoimmunity, and negative tests for viral hepatitis. Twenty cases were divided into AIH $(n=12)$ or AI-DILI $(n=8)$ groups. Blind qualitative evaluation of necroinflammatory changes and liver fibrosis were performed according to the Scheuer scoring system. Cellular densities were determined using Image] (V1.51t, National Institutes of Health, Bethesda, MD, USA). Fibrosis was assessed on Masson trichrome-stained slides, and collagen deposition was estimated following a protocol of color deconvolution. Results: Necroinflammatory changes as well as densities (portal and lobular) of neutrophils and eosinophils, intracellular cholestasis, and regenerative changes did not differ between the two groups $(P \geq 0.05)$. Neutrophil densities but not eosinophils showed a positive correlation with the severity of hepatocellular damage $(r=0.6$ and 0.58 , vs. alanine aminotransferase, $P<0.05$ ). Ceroidladen macrophages but not histiocytic aggregates appeared to be more common in AI-DILI $(P<0.05)$. AIH patients presented more often with evidence of chronic damage, including higher scores of fibrosis and collagen deposition, in comparison to AI-DILI $(P<0.05)$. Conclusions: Although there is no histologic feature pathognomonic for AI-DILI or AIH, advanced stages of liver fibrosis can be used to support the diagnosis of AIH in some cases. Definitive diagnosis of AI-DILI requires follow-up and demonstration of complete remission after drug withdrawal with no need for immunosuppression.
\end{abstract}

Citation of this article: Febres-Aldana CA, Alghamdi S, Krishnamurthy K, Poppiti RJ. Liver fibrosis helps to distinguish autoimmune hepatitis from DILI with autoimmune

Keywords: DILI; Autoimmune hepatitis; Liver fibrosis; Digital pathology. Abbreviations: AIH, idiopathic autoimmune hepatitis; AI-DILI, DILI with autoimmune features; ALT, alanine aminotransferase; DILI, drug-induced liver injury. Received: 28 September 2018; Revised: 15 November 2018; Accepted: 23 November 2018

*Correspondence to: Christopher A. Febres-Aldana, Mount Sinai Medical Center, Department of Pathology and Laboratory Medicine, 4300 Alton Rd, Miami Beach, FL 33140, USA. Tel: +1-305-674-2277, Fax: +1-305-674-2999, E-mail: christopher.febres@msmc.com features: A review of twenty cases. J Clin Transl Hepatol 2019;7(1):21-26. doi: 10.14218/JCTH.2018.00053.

\section{Introduction}

Autoimmune hepatitis consists of immune-mediated damage of hepatocytes associated with the development of autoantibodies. ${ }^{1}$ The diagnoses of idiopathic autoimmune hepatitis (AIH) and drug-induced liver injury (DILI) are challenging because both conditions show overlapping manifestations. Recently, DILI with autoimmune features (AI-DILI) has been recognized as a discrete entity. ${ }^{2}$ AI-DILI is characterized by liver injury due to the ingestion of medications or herbal products with simultaneous positivity for markers of liver autoimmunity (high IgG immunoglobulin levels, antinuclear antibodies, anti-smooth muscle antibodies, anti-liver-kidney microsomal antibodies, and rarely anti-mitochondrial antibodies). The liver damage becomes clinically evident within 3 months following the drug exposure, but it can appear after a longer latency interval. ${ }^{2,3}$ Among the medications causing this syndrome are statins, diclofenac, hydralazine, methyldopa, minocycline, nitrofurantoin, and procainamide. ${ }^{3}$

By definition, in AI-DILI, the liver injury must completely resolve after drug withdrawal with no recurrence. Recovery is reached either spontaneously or with immunosuppressive therapy. Steroids and azathioprine are among the medications applied to treat AI-DILI. 2,3 Time to resolution varies from days to weeks, lasting months for a complete normalization of liver enzymes in a few cases. Those cases that relapse after drug withdrawal have been described as $\mathrm{AIH}$ triggered by drugs. Furthermore, relapse after immunosuppressive therapy withdrawal does not occur in AI-DILI, and its absence distinguishes AI-DILI from classical AIH. ${ }^{4,5}$ Timely diagnosis is critical for proper management in both conditions. Early immunosuppression can lead to remission in AIH. Likewise, prompt identification and discontinuation of toxic drugs halt liver injury in AI-DILI. Failure to properly treat AIH or AI-DILI could result in adverse clinical outcomes. ${ }^{3}$

AIH can present with typical histopathological findings, but DILI may mimic any non-DILI pattern of liver injury, including $\mathrm{AIH}$. Critical characteristics of AIH include a mixed inflammatory infiltrate composed of lymphocytes and plasma cells that is most marked around portal areas and is referred to as interface hepatitis. The presence of a predominance of plasma cells within the infiltrate is highly suggestive of the diagnosis. Necrosis of periportal hepatocytes, scattered acidophil or apoptotic bodies, and cytoplasmic swelling are 
Febres-Aldana C.A. et al: Fibrosis in autoimmune hepatitis vs DILI

manifestations of hepatocellular damage. AIH may also cause the full temporal spectrum of liver injury, from mild fibrosis to established cirrhosis. ${ }^{1}$ Diagnosing AIH also requires ruling out infectious causes of liver injury, including viral hepatitides. ${ }^{6}$ AI-DILI mimics the morphological pattern of $\mathrm{AIH}$, including the prominent lymphoplasmacytic infiltrates in portal spaces and interface hepatitis. ${ }^{2,7}$ In this study, we performed a histopathological evaluation of liver biopsies to further identify potential hallmarks for differentiating both entities.

\section{Methods}

\section{Case selection}

Liver biopsies stored in the tissue archives of Mount Sinai Medical Center Department of Pathology for the past 10 years were reviewed. Cases were included if there was no history of prior liver disease and when the primary clinical and pathological differential diagnosis was $\mathrm{AIH}$, according to recommendations of the American Association for the Study of Liver Diseases. ${ }^{4}$ The diagnosis of AIH was based on the presence of the autoantibodies anti-smooth muscle antibodies or antiliver-kidney microsomal antibodies, or high IgG levels with compatible histology and exclusion of infectious etiologies. All cases had to show predominant lymphoplasmacytic infiltrates with piecemeal necrosis (Fig. 1). Sorting into the AIH or AI-DILI groups was done retrospectively according to pertinent clinical history, follow-up notes, and laboratory tests supporting liver autoimmunity and ruling out viral hepatitis.
Liver biopsies included in the analysis were those performed at the time of presentation, when no diagnosis was known. For AI-DILI, the liver injury had to be associated with a drug exposure and completely resolve after drug withdrawal and with no need for immunosuppression in subsequent follow-ups. If a patient developed a relapse or persistent liver damage after recovery from a drug exposure, the case was included in the AIH group. Charts were reviewed to rule out re-challenge to the hepatotoxic drug. Relapsing cases of AIH triggered by drugs must not have been exposed again to the suspected drug in order to be considered part of this group. The Mount Sinai Medical Center Institutional Review Board approved the study in conformance to the ethical guidelines of the 1975 Declaration of Helsinki.

\section{Histological evaluation}

Blind qualitative evaluation of necroinflammatory changes and fibrosis were graded according to the Scheuer scoring system. ${ }^{8}$ Presence or absence of intracellular cholestasis, ceroid-laden macrophages, and histiocytic aggregates were also recorded (Fig. 1). Regenerative changes and hepatocyte necrosis were evaluated in reticulin-stained slides. Cellular densities were determined on captured images using Image] (V1.51t, National Institutes of Health, Bethesda, MD, USA). Liver fibrosis was assessed on Masson trichrome-stained slides, and collagen deposition was estimated on captured images following a protocol of color deconvolution. 9,10 The amount of collagen was recorded as a fraction area of the adjusted threshold value of green color within the region of interest (Fig. 2a).

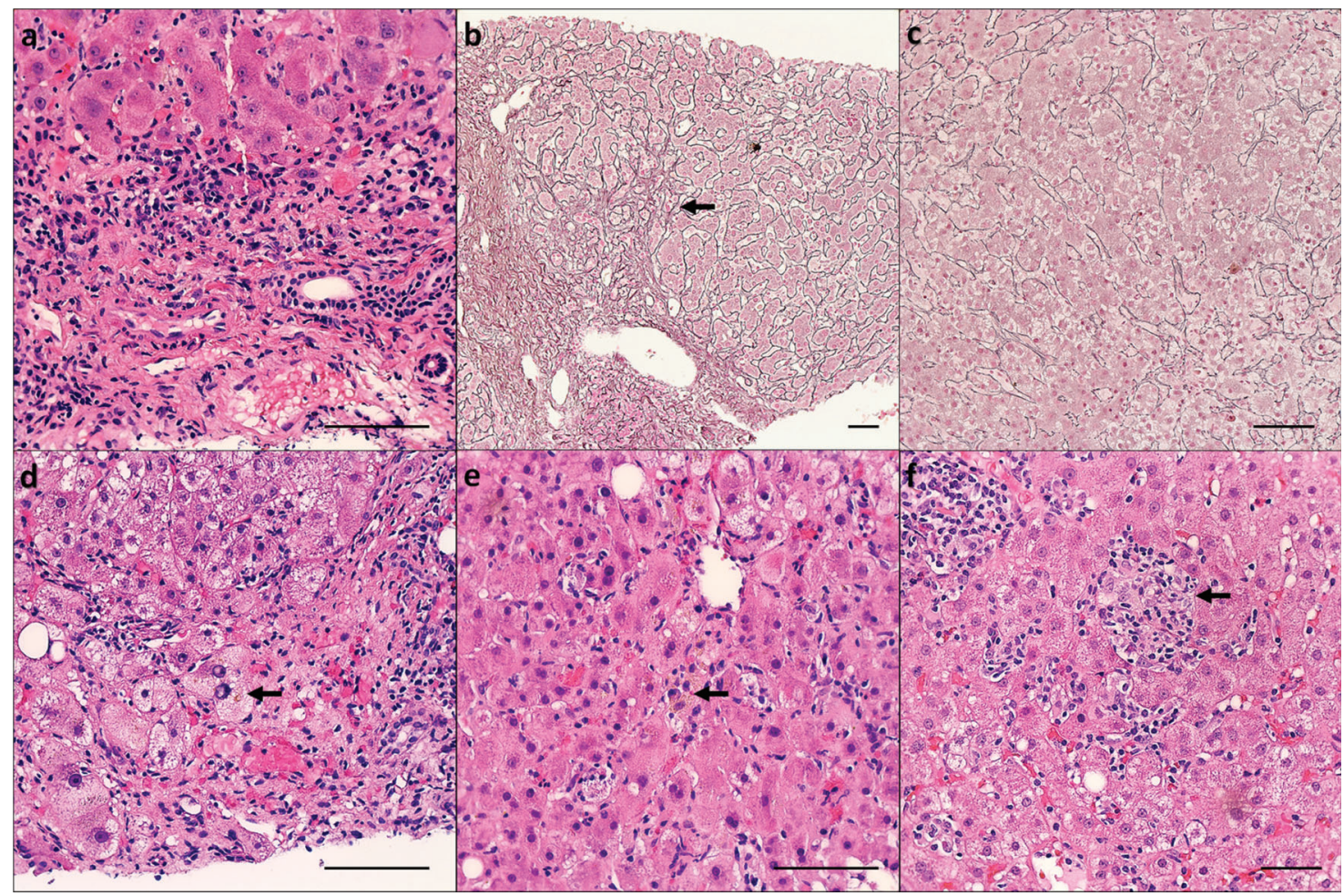

Fig. 1. Histopathologic features of AIH and AI-DILI. (a) Interface hepatitis with a portal lymphoplasmacytic infiltrate, H\&E. (b) Collapse of the peri-hepatocyte reticulin network for grading necrosis (arrow) in difficult cases, reticulin stain. (c) Hepatocyte regeneration identified by distension of trabeculae, reticulin stain. (d) Intracellular (hepatocellular) cholestasis (arrow), H\&E. (e) Ceroid-laden macrophages (arrow), H\&E. (f) Collection of histiocytes (arrow), H\&E. Bar: 100 $\mu$ m. Abbreviation: H\&E, hematoxylin and eosin. 
Febres-Aldana C.A. et al: Fibrosis in autoimmune hepatitis vs DILI

\section{Statistical analysis}

Scheuer scores and other histologic features were compared using Wilcoxon rank-sum and Fisher's exact tests. Cellular densities and fraction areas of collagen deposition were compared using multiple or unpaired t-test. Correlation analysis was performed using Pearson or Spearman tests depending on the type of variable. All $P$ values presented were two-sided and considered statistically significant when less than 0.05. The statistical analysis was performed in Microsoft Excel ${ }^{\circledR}$ (V15; Microsoft Corporation, Redmond, WA, USA) and GraphPad Prism ${ }^{\circledR}$ (V6; GraphPad Software Inc, La Jolla, CA, USA).

\section{Results}

\section{Patient features}

The total number of cases was 20. Detailed patient's characteristics including history of autoimmune diseases, evidence of liver autoimmunity at presentation, latency period, and recovery time are shown in Table 1 . There were no significant differences between the AIH $(n=12)$ and AI-DILI $(n=8)$ groups regarding age (49 [24-66] vs. 54 [17-71], median [range], respectively). The male gender was relatively more frequent in AIH than in AI-DILI (33\% vs. $13 \%$, respectively). The mean \pm SEM of laboratory liver parameters at presentation were not significantly different between the AIH and AI-DILI groups: $938.9 \pm 281.9$ versus $1339 \pm 395.7 \mathrm{U} / \mathrm{L}$ for alanine aminotransferase (ALT) levels; $1084 \pm 337.2$ versus
836.7 $\pm 257.7 \mathrm{U} / \mathrm{L}$ for aspartate aminotransferase levels; $187 \pm 26.01$ versus $299.8 \pm 114.8 \mathrm{U} / \mathrm{L}$ for alkaline phosphatase levels; and $7.41 \pm 3.001$ versus $4.58 \pm 1.54 \mathrm{mg} / \mathrm{dL}$ for total bilirubin levels ( $P \geq 0.05$, unpaired $t$-test). Exposure to a drug known to cause AI-DILI was documented in 33\% (4/12) of $\mathrm{AIH}$ cases and $100 \%(8 / 8)$ of AI-DILI. Drugs of the AIH group included clavulanic acid, levofloxacin, ramipril, and simvastatin. Doxycycline, atorvastatin, simvastatin, ciprofloxacin, lisinopril, isoniazid, OxyELITE, and ustekinumab were identified in the AI-DILI group. The mean follow-up period for both groups was 57 months (12-108, range).

\section{Patients with AIH presented with a higher degree of liver fibrosis than those with AI-DILI}

According the Scheuer scoring system (Fig. 2b), 8 out of 20 patients presented with no liver fibrosis (score: 0), 5 out of 20 patients with enlarged fibrotic portal tracts (score: 1), 7 out of 20 patients with higher grades of liver fibrosis (scores: 2, 3, and 4). The amount of collagen deposition shows a strong positive correlation with Scheuer stages of liver fibrosis $(r=0.9201, P<0.0001$, Spearman correlation; Fig. 2b). The mean Scheuer score for liver fibrosis in AIH was $1.583 \pm 1.165$ versus $0.375 \pm 0.7440$ in AI-DILI (mean \pm SD, $P<0.01$, Wilcoxon rank-sum test; Fig. 2c). Advanced stages of liver fibrosis ( 3 and 4 ) were seen in only two patients with AIH. Likewise, the amount of collagen deposition was more prominent in AIH than AI-DILI (14.83 \pm 3.775 vs. 4.205 \pm 1.3565 , mean \pm SEM, $P<0.05$, unpaired t-test; Fig. 2d).

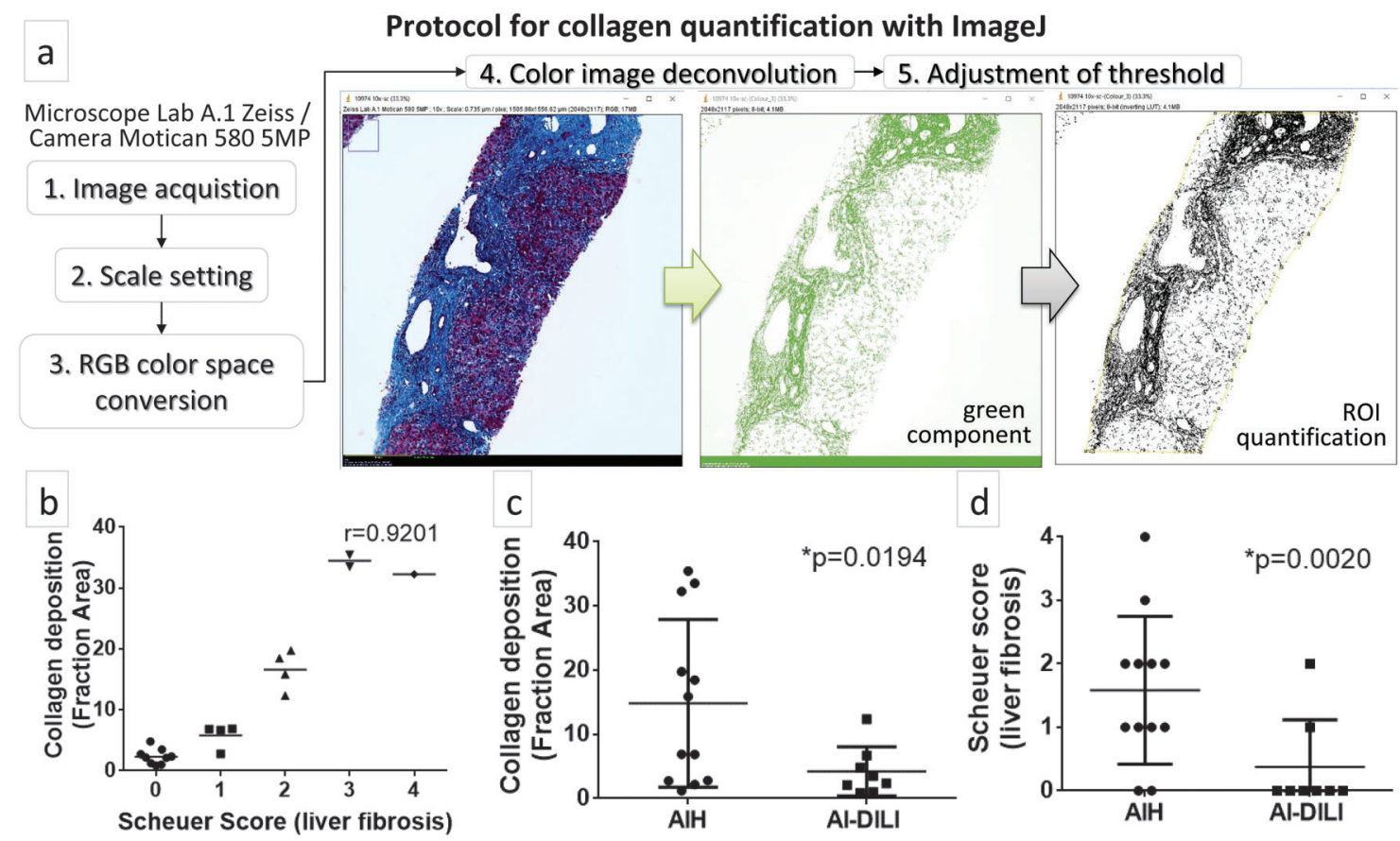

Fig. 2. Evaluation of liver fibrosis in AIH versus AI-DILI. Digital imaging protocol using the Image] software (5) was applied to quantify collagen deposition in liver biopsies (a). Color deconvolution of captured trichrome Masson-stained images allows separation of collagen (green component) from the background. The threshold was manually adjusted until the entire green area was highlighted in red, then converted in gray/black color. The amount of collagen was measured as fraction area-based quantification in the regions of interest (ROI). Collagen deposition determined by this method shows a strong correlation with the qualitative Scheuer scoring system for liver fibrosis (b) (mean, $r=0.9201$, Spearman correlation). Patients with AIH were more likely to present with higher degrees of liver fibrosis than those with AI-DILI, as shown by collagen deposition (c, mean \pm SD, unpaired $t$-test) and Scheuer scores (d, mean \pm SD, Wilcoxon rank-sum test). Fibrosis in AIH is a consequence of chronicity of liver damage, a condition that is diagnosed during flares of disease, while AI-DILI more likely presents as (sub)acute onset cases with minimal or no fibrosis. 
Febres-Aldana C.A. et al: Fibrosis in autoimmune hepatitis vs DILI

Table 1. Caractheristics of patients with AIH and AI-DILI

\begin{tabular}{|c|c|c|c|c|c|c|c|c|}
\hline Case & Age & Gender & $\begin{array}{l}\text { Evidence of liver } \\
\text { autoimmunity }\end{array}$ & $\begin{array}{l}\text { History of } \\
\text { autoimmune } \\
\text { disease }\end{array}$ & $\begin{array}{l}\text { Suspected } \\
\text { drug }\end{array}$ & $\begin{array}{l}\text { Latency } \\
\text { period }\end{array}$ & $\begin{array}{l}\text { Recovery } \\
\text { period }\end{array}$ & $\begin{array}{l}\text { Liver } \\
\text { fibrosis at } \\
\text { presentation* }\end{array}$ \\
\hline \multicolumn{9}{|c|}{ AI-DILI cases } \\
\hline 1 & 71 & $\mathrm{~F}$ & anti-SM & None & Doxycycline & 20 days & 3 months & 2 \\
\hline 2 & 53 & $\mathrm{~F}$ & anti-SM; ANA & None & OxyELITE & 2 months & 12 months & 0 \\
\hline 3 & 17 & M & anti-SM & None & Doxycycline & 14 days & 6 months & 0 \\
\hline 4 & 81 & $\mathrm{~F}$ & Increased IgG & None & Symvastatin & 12 months & 6 months & 1 \\
\hline 5 & 27 & $\mathrm{~F}$ & $\begin{array}{l}\text { Anti-LKM; anti-SM; } \\
\text { increased IgG }\end{array}$ & None & Ciprofloxacin & 5 days & 30 days & 0 \\
\hline 6 & 65 & $\mathrm{~F}$ & Increased IgG; ANA & None & Lisinopril & 1 month & 3 months & 0 \\
\hline 7 & 63 & $\mathrm{~F}$ & $\begin{array}{l}\text { Anti-LKM; anti-SM; } \\
\text { increased IgG }\end{array}$ & None & Atorvastatin & 4 months & 9 months & 0 \\
\hline 8 & 54 & $\mathrm{~F}$ & $\begin{array}{l}\text { Increased IgG; } \\
\text { anti-SM }\end{array}$ & Psoriasis & Ustekinumab & 30 days & 6 months & 0 \\
\hline \multicolumn{9}{|c|}{ AIH cases } \\
\hline 9 & 36 & $\mathrm{~F}$ & Anti-SM & None & Clavulanic acid & 7 days & N/A & 2 \\
\hline 10 & 30 & M & Increased IgG & None & None & N/A & N/A & 1 \\
\hline 11 & 54 & M & Anti-LKM; anti-SM & $\mathrm{HT}$ & None & $\mathrm{N} / \mathrm{A}$ & N/A & 0 \\
\hline 12 & 51 & $M$ & Increased IgG & None & Ramipril & 12 months & N/A & 0 \\
\hline 13 & 62 & $\mathrm{~F}$ & Increased IgG & None & Levofluoxacin & 7 days & N/A & 4 \\
\hline 14 & 63 & $\mathrm{~F}$ & $\begin{array}{l}\text { Increased IgG; } \\
\text { anti-SM }\end{array}$ & None & None & $\mathrm{N} / \mathrm{A}$ & N/A & 3 \\
\hline 15 & 66 & M & Increased IgG & None & None & N/A & N/A & 2 \\
\hline 16 & 53 & $\mathrm{~F}$ & Anti-SM & None & None & N/A & N/A & 1 \\
\hline 17 & 36 & $\mathrm{~F}$ & Increased IgG & SLE & None & N/A & N/A & 1 \\
\hline 18 & 60 & $\mathrm{~F}$ & $\begin{array}{l}\text { Increased IgG; } \\
\text { anti-SM }\end{array}$ & None & Symvastatin & 8 months & N/A & 2 \\
\hline 19 & 24 & $\mathrm{~F}$ & $\begin{array}{l}\text { Anti-LKM; anti-SM; } \\
\text { increased IgG; ANA }\end{array}$ & None & None & N/A & N/A & 1 \\
\hline 20 & 39 & $\mathrm{~F}$ & Anti-LKM; anti-SM & AS & None & N/A & N/A & 2 \\
\hline
\end{tabular}

Latency period: symptom onset after drug exposure; Recovery: time normal liver tests.

* Scheuer scores: 0 , none; 1 , enlarged fibrotic portal tracts; 2 , periportal or portal-portal septa but intact architecture; 3 , fibrosis with architectural distorsion but no obvious cirrhosis; 4, probable cirrhosis.

Abbreviations: ANA, antinuclear antibodies; Anti-LKM, anti-liver-kidney microsomal; Anti-SM, anti-smooth muscle; AS, antiphospholipid syndrome; F, female; HT, Hashimoto thyroiditis; LKM, liver-kidney microsomal; M, male; N/A, not applicable; SLE, systemic lupus erythematous; SM: smooth muscle.

\section{The degree of necroinflammatory changes correlates with the severity of hepatocellular damage but not with the etiology, in both groups}

The severity of the inflammatory response, determined by Scheuer score (0-4), did not differ between patients with AIH versus AI-DILI in either portal spaces $(2.083 \pm 0.996$ vs. $2.125 \pm 0.64)$ or hepatic lobules $(1.917 \pm 1.084$ vs. 1.875 \pm 0.991 ) (mean $\pm S D, P \geq 0.05$, Wilcoxon rank-sum test). The cellular density of neutrophils and eosinophils in hotspots of portal spaces and hepatic lobules was also similar in both groups (mean $\pm \mathrm{SD}, P \geq 0.05$, multiple $t$-test using the SidakBonferroni method; Fig. 3a). Moreover, the cellular density of neutrophils in portal spaces and lobules, but not eosinophils, showed a positive correlation with the severity of hepatocellular damage in both groups, as measured by ALT levels $(r=0.608$ vs. neutrophil density in portal spaces, $P<0.05$; $r=0.586$ vs. neutrophil density in hepatic lobules, $P<0.05$ $r=0.209$ vs. eosinophil density in portal spaces, $P \geq 0.05$; $r=0.338$ vs. eosinophil density in hepatic lobules, $P \geq 0.05$, Pearson correlation; Fig. 3b). The density of neutrophils in lobules but not portal spaces showed a positive correlation with necroinflammatory scores by the Scheuer method ( $r=0.5766, P<0.01$, in lobules and $r=0.3236, P \geq 0.05$, in portal spaces, Spearman correlation).

\section{The presence of ceroid-laden macrophages appears to be more common in AI-DILI and histiocytic aggregates in AIH}

The presence or absence of additional histologic features of liver damage was also assessed and showed that ceroidladen macrophages were more common in AI-DILI (87.5\% vs. $33.3 \%$ in $\mathrm{AIH}, P<0.05$, Fisher's exact test). Histiocytic 
Febres-Aldana C.A. et al: Fibrosis in autoimmune hepatitis vs DILI
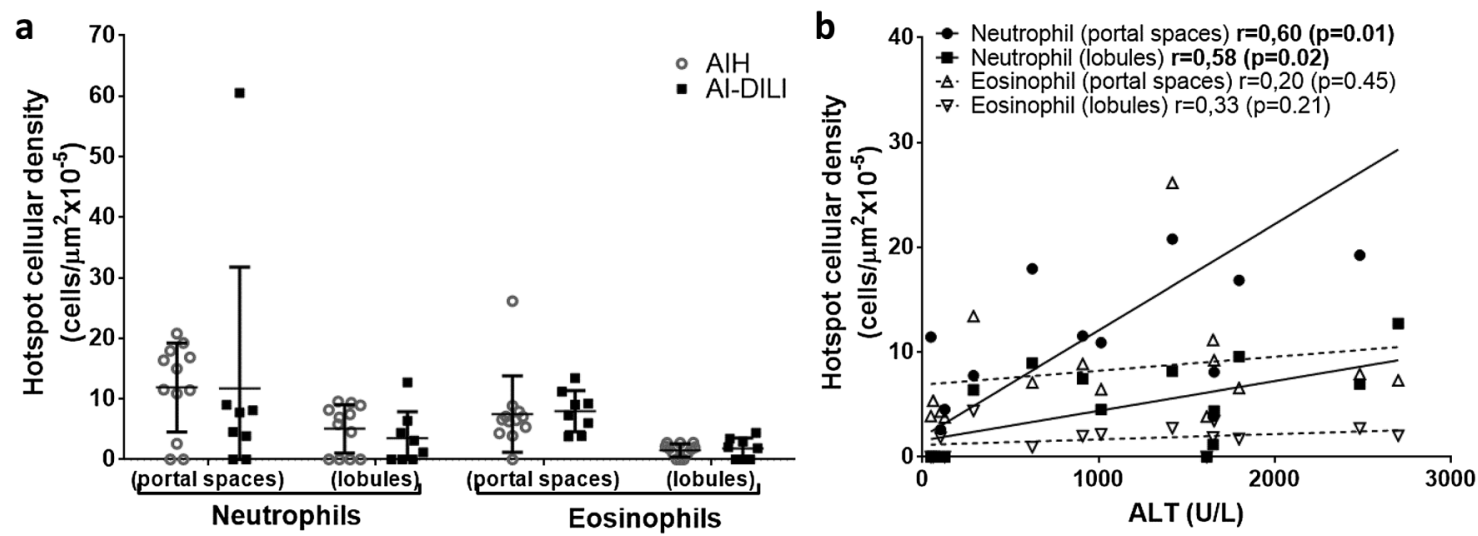

Fig. 3. Cellular densities and distribution of neutrophils and eosinophils in AIH versus AI-DILI. The amounts of neutrophils and eosinophils in portal spaces and hepatic lobules (cells $/ \mu \mathrm{m}^{2} \times 10^{-5}$ ) did not differ between AIH (white circle) and AI-DILI (black square), (a, mean $\pm \mathrm{SD}, P \geq 0.05$, multiple $t$-test using the Sidak-Bonferroni method). Instead, the cellular density of neutrophils (black circle: portal spaces; black square: hepatic lobules) but not eosinophils (upward white triangle: portal spaces; downward white triangle: hepatic lobules), correlates with severity of hepatocellular damage measured by ALT levels (U/L) in both groups, (b, solid and dashed lines, simple linear regression for neutrophil and eosinophil densities vs. ALT levels, $r$ and $P$ values for Pearson correlation analysis).

aggregates were more common in $\mathrm{AIH}$ ( $75 \%$ vs. $37.5 \%$ in AI-DILI, $P<0.05$, Fisher's exact test). The frequency of intracellular cholestasis $(62.5 \%$ in AI-DILI vs. $33.3 \%$ in $\mathrm{AIH})$ and regenerative changes $(12.5 \%$ in AI-DILI vs. $50 \%$ in $\mathrm{AIH}$ ) were not statistically different between groups $(P \geq 0.05$, Fisher's exact test).

\section{Discussion}

The etiology of autoimmune liver disease is unknown, for the most part. The occurrence of liver autoantibodies can even be detected in infectious hepatitides, which suggest that they are rather nonspecific and should be used with caution when diagnosing $\mathrm{AIH} .{ }^{11}$ There are reports of drug-induced hepatotoxicity accompanied by an autoimmune response. For that reason, AI-DILI is a differential diagnosis of AIH in daily practice. ${ }^{2}$ Histopathology of liver biopsies has been useful in diagnosing DILI and AIH. However, a significant problem for hepatologists and pathologists comes when separating AIH from AI-DILI as both entities share clinical, biochemical, and histopathologic features. ${ }^{1-3}$ Patients with new-onset AIH frequently report recent use of medications that are also associated with DILI, such as antibiotics, statins or antihypertensive drugs. ${ }^{1-3,12}$ We aimed in this study to identify histopathological features that can help separate AIH from AI-DILI.

Microscopic evaluation showed comparable severity in interface hepatitis, hepatocyte necrosis, portal and lobular inflammation, and infiltration of eosinophils in both conditions. In a previous study, the presence of cholestasis and portal neutrophils favored AI-DILI over $\mathrm{AIH}^{7}$ Our results suggest that infiltration of neutrophils positively correlates with the severity of hepatocellular damage but not with the etiology. Intracellular cholestasis seemed to be more frequent in AI-DILI and regenerative changes in $\mathrm{AIH}$, but they were not statistically significant. Ceroid-laden macrophages were more common in AI-DILI, and prominent histiocytic infiltrates were mainly seen in $\mathrm{AIH}$; however, none of those features were skewed enough to be useful as a marker to differentiate AI-DILI from AIH in this small series. The full spectrum of necroinflammatory changes that can develop in the context of liver autoimmunity was also observed in AI-DILI.
Historically, liver infiltration of eosinophils has been used to identify cases of drug hypersensitivity, allergic diseases, malignancies, hyper-eosinophilic syndrome, collagen vascular diseases, and, most commonly, parasitic infections. However, we found that eosinophils are observed in liver biopsies in patients with $\mathrm{AIH}$. In terms of quantity, some forms of DILI (particularly the immunoallergic-type) are accompanied by copious amounts of eosinophils undoubtedly greater than what is seen in a typical case of $\mathrm{AIH} .{ }^{13}$ However, AI-DILI is a distinct and rare entity among DILI reactions that appears to be mediated by immune mechanisms very similar to AIH. Thus, the density of eosinophils is not expected to be quite different between AI-DILI and AIH. We excluded other forms of DILI in this study, which may also explain that result. The inclusion criteria was very strict, and evidence of liver autoimmunity must have been documented in the chart for all cases. Liver infiltration of eosinophils has diagnostic value for some liver diseases but does not appear to help in the distinction of AI-DILI versus AIH.

Interestingly, owing to the chronic nature of $\mathrm{AIH}$, persistent loss of hepatocytes leads to progressive fibrosis and eventually cirrhosis. In this way, the presence of advanced stages of liver fibrosis could be used clinically to favor the diagnosis of AIH over AI-DILI in patients with no history of a previously diagnosed chronic liver disease. This proposal was demonstrated by the presence of higher Scheuer scores of liver fibrosis and abundant collagen deposition in patients with AIH. The Scheuer scoring system has been commonly used in grading viral hepatitis-associated chronic damage and has shown good reproducibility. ${ }^{8}$ The protocol of color deconvolution applied to quantify collagen had not been validated for staging liver fibrosis; however, we found an excellent correlation between Scheuer scores and collagen deposition measurements. Fibrosis in the AIH group represents a marker of chronicity of disease. By the time a patient with AIH develops significant symptoms, it may be the expression of acute-onset $\mathrm{AIH}$ or a flare of previously unrecognized AIH. Therefore, significant liver fibrosis detected on liver biopsies can be used to favor a diagnosis of AIH, regardless of whether or not it is considered acute-onset AIH. ${ }^{12}$ 
Febres-Aldana C.A. et al: Fibrosis in autoimmune hepatitis vs DILI

Early stages of liver fibrosis can be seen in AI-DILI. It is unclear whether mild fibrosis in AI-DILI is a consequence of prolonged drug toxicity or a different unrecognized liver insult. Liver fibrosis in many diseases, including $\mathrm{AIH}$, is a progressive phenomenon and takes years to develop. ${ }^{14}$ Furthermore, drug toxicity in AI-DILI has been documented within 3 months before clinically detectable liver damage, which makes any degree of fibrosis unlikely to be secondary to drug toxicity. In a prior study, cirrhosis was only observed in AIH cases, whereas no cirrhosis was present among AI-DILI cases. ${ }^{7}$ Although not all AI-DILI cases are acute or AIH cases chronic, AI-DILI usually causes liver damage severe enough to produce significant symptoms, leading to an early diagnosis. In many patients with $\mathrm{AIH}$, the liver injury tends to be subclinical with waxing and waning episodes. AIH is detected during work-ups of long-standing unexplained increased liver enzymes or severe acute episodes of liver injury. Cases of late-onset AI-DILI, presenting at 1-2 years after drug exposure, are uncommon but possible, being more frequent with minocycline and nitrofurantoin. Advanced liver fibrosis and cirrhosis is absent in those cases of late-onset AI-DILI cases. ${ }^{2}$ In a series of 24 patients with AI-DILI, none of them presented with cirrhosis or significant fibrosis at baseline. ${ }^{15}$ Our findings are consistent with the fact that advanced fibrosis (i.e. marked bridging fibrosis) was observed mainly among AIH cases, but not AI-DILI.

Our study has limitations. First, our sample size is small, and its statistical power was not sufficient for the analysis of some histopathological features that are likely to be diagnostically useful in conjunction by multivariate analysis. The follow-up in some patients was short, and progression to a fully developed AIH phenotype cannot be completely ruled out. We limited the assessment of histological features to variables with high reproducibility among pathologists. Features such as the so-called hepatocyte rosette and emperipolesis are difficult to evaluate and lack diagnostic value for AIH. ${ }^{16}$ For that reason, they were not included in this study.

\section{Conclusions}

In summary, AI-DILI exhibits the clinical and pathologic features of AIH. Necroinflammatory changes, and infiltration of neutrophils or eosinophils are manifestations of hepatocellular damage, and are nonspecific findings. There is no individual histopathologic feature decisive for diagnosing AI-DILI over AIH. AIH can cause the full spectrum of liver injury, from mild fibrosis to established cirrhosis. Advanced stages of liver fibrosis can be used to favor the diagnosis of AIH over AI-DILI in patients with no history of liver disease. Definitive diagnosis of cases presenting with autoimmune liver injury and mild liver fibrosis must be made by follow-up. AI-DILI requires demonstration of complete remission after drug withdrawal and no need for continuous immunosuppression.

\section{Conflict of interest}

The authors have no conflict of interests related to this publication.

\section{Author contributions}

Designed the study (CAF, RJP), wrote the manuscript, designed the figures and table, reviewed the medical records and performed the statistical analyses (CAF), performed the pathologic evaluation of liver biopsies (CAF, SA, KK, RJP). All authors drafted and approved the final version of this manuscript.

\section{References}

[1] Webb G], Hirschfield GM, Krawitt EL, Gershwin ME. Cellular and molecular mechanisms of autoimmune hepatitis. Annu Rev Pathol 2018;13:247-292. doi: 10.1146/annurev-pathol-020117-043534.

[2] deLemos AS, Foureau DM, Jacobs C, Ahrens W, Russo MW, Bonkovsky HL. Drug-induced liver injury with autoimmune features. Semin Liver Dis 2014 34:194-204. doi: 10.1055/s-0034-1375959.

[3] de Boer YS, Kosinski AS, Urban T], Zhao Z, Long N, Chalasani N, et al. Features of autoimmune hepatitis in patients with drug-induced liver injury. Clin Gastroenterol Hepatol 2017;15:103-112.e2. doi: 10.1016/j.cgh.2016.05.043.

[4] Heurgué-Berlot A, Bernard-Chabert B, Diebold MD, Thiéfin G. Drug-induced autoimmune-like hepatitis: a case of chronic course after drug withdrawal. Dig Dis Sci 2011;56:2504-2505; author reply 2505. doi: 10.1007/s10620011-1786-8.

[5] Czaja AJ. Drug-induced autoimmune-like hepatitis. Dig Dis Sci 2011;56: 958-976. doi: 10.1007/s10620-011-1611-4.

[6] Manns MP, Czaja AJ, Gorham JD, Krawitt EL, Mieli-Vergani G, Vergani D, et al. Diagnosis and management of autoimmune hepatitis. Hepatology 2010;51: 2193-2213. doi: 10.1002/hep.23584.

[7] Suzuki A, Brunt EM, Kleiner DE, Miquel R, Smyrk TC, Andrade RJ, et al. The use of liver biopsy evaluation in discrimination of idiopathic autoimmune hepatitis versus drug-induced liver injury. Hepatology 2011;54:931-939. doi: $10.1002 /$ hep. 24481.

[8] Scheuer PJ. Classification of chronic viral hepatitis: a need for reassessment. J Hepatol 1991;13:372-374. doi: 10.1016/0168-8278(91)90084-O.

[9] Ruifrok AC, Johnston DA. Quantification of histochemical staining by color deconvolution. Anal Quant Cytol Histol 2001;23:291-299.

[10] Chen Y, Yu Q, Xu CB. A convenient method for quantifying collagen fibers in atherosclerotic lesions by Image] software. Int J Clin Exp Med 2017;10: 14904-14910.

[11] Ghonaim M, Al-Ghamdi A, El-Bana H, Bakr A, Ghoneim E, El-Edel R, et al. Autoantibodies in chronic liver disease. Egypt J Immunol 2005;12:101-111.

[12] Fujiwara K, Yasui S, Tawada A, Fukuda Y, Nakano M, Yokosuka O. Diagnostic value and utility of the simplified International Autoimmune Hepatitis Group criteria in acute-onset autoimmune hepatitis. Liver Int 2011;31:1013-1020. doi: 10.1111/j.1478-3231.2011.02524.x.

[13] Kleiner DE, Chalasani NP, Lee WM, Fontana RJ, Bonkovsky HL, Watkins PB, et al. Hepatic histological findings in suspected drug-induced liver injury: systematic evaluation and clinical associations. Hepatology 2014;59:661670. doi: 10.1002/hep.26709.

[14] Hernandez-Gea V, Friedman SL. Pathogenesis of liver fibrosis. Annu Rev Pathol 2011;6:425-456. doi: 10.1146/annurev-pathol-011110-130246.

[15] Björnsson E, Talwalkar J, Treeprasertsuk S, Kamath PS, Takahashi N, Sanderson $\mathrm{S}$, et al. Drug-induced autoimmune hepatitis: clinical characteristics and prognosis. Hepatology 2010;51:2040-2048. doi: 10.1002/hep.23588.

[16] Balitzer D, Shafizadeh N, Peters MG, Ferrell LD, Alshak N, Kakar S. Autoimmune hepatitis: review of histologic features included in the simplified criteria proposed by the international autoimmune hepatitis group and proposal for new histologic criteria. Mod Pathol 2017;30:773-783. doi: 10. 1038/modpathol.2016.267. 